Aim of the study: Fallopian tube cancer is very rare in the literature and so there are not enough data about the therapeutic approaches. The approaches are generally determined in accordance with the data obtained from ovarian cancer. Many prognostic factors have been investigated in an effort to better estimate patient outcome. Stage, age, and residual tumor after surgery are consistently important prognostic factors. In this study, we aimed to evaluate the prognostic factors and survival rates of primary fallopian tube cancer (PFTC), which is rare among gynecological cancers.

Material and methods: Thirty-eight patients with a diagnosis of PFTC were identified through the gynecologic oncology service database of our Research and Training Hospital in the period 1995-2013. Clinicopathological and surgical data were collected. All patients were evaluated for survival and disease-free survival between the dates specified.

Results: A significant relationship and correlation was found between optimal surgery and life expectancy. Better results were obtained in patients treated with optimal surgery. The survival probability was found to be higher in patients with lower CA-125 levels and serous histologic type adenocarcinoma.

Conclusions: Stage is one of the factors affecting the survival probability. We determined that the pathological type of tumor, the diameter of residual tumor remaining after surgery, tumor grade, preoperative CA- 125 levels and presence of ascites affect the survival probability.

Key words: primary fallopian tube cancer, prognostic factor, management and treatment.

Contemp Oncol (Pozn) 2018; 22 (2): 99-104 DOI: https://doi.org/10.5114/wo.2017.69590

\section{Prognostic factors of primary fallopian tube carcinoma}

\author{
Emrah Akkaya ${ }^{1}$, Muzaffer Sanci ${ }^{1}$, Nur G. Kulhan², Mehmet Kulhan ${ }^{2}$, \\ Umit Nayki², Cenk Nayki², Nahit Ata², Pasa Ulug ${ }^{2}$
}

${ }^{1}$ Obstetrics and Gynaecology Unit, Tepecik Obstetrics and Gynaecology Training and Research Hospital, Izmir, Turkey

${ }^{2}$ Department of Obstetrics and Gynecology, Erzincan University, School of Medicine, Turkey

\section{Introduction}

Primary fallopian tube carcinoma (PFTC) is a rare neoplasm, accounting for $0.3-1.6 \%$ of all gynecological malignancies [1, 2]. Because of the fallopian tube neighborhood, secondary fallopian tumors are more commonly seen. Due to its clinical and histologic similarity to ovarian cancers, PFTC is treated as ovarian cancer. Primary fallopian tube carcinoma can show sequential growth or it may be associated with other cancers. It has been reported that $25 \%$ of the cases have another cancer before PFTC and $22 \%$ of the cases have another gynecological cancer simultaneously [3].

Primary fallopian tube carcinoma was described for the first time in 1847 by Rokitansky and in 1861 by Renaud. It usually occurs between 50 and 60 years of age. The youngest patient reported in the literature is 19 years old, the oldest 87 years old. The mean age is between 52.7 and 57. Approximately $90 \%$ of PFTCs are adenocarcinomas and most of them are serous and endometrioid types [1, 2].

The spread pattern is similar to ovarian cancer. Primary fallopian tube carcinoma spreads very quickly, so the number of patients diagnosed early is very small. Generally, the diagnosis is made during operations performed for other reasons. It is most commonly spread by intraperitoneal and lymphoid routes. However, unlike ovarian cancer, PFTC has higher rates of retroperitoneal and distant metastases [4]. It has no obvious symptoms and $20 \%$ of patients are asymptomatic. The most common symptom is vaginal bleeding. Fifty percent of patients have vaginal bleeding. Discharge or bleeding is accompanied by pain in $26-50 \%$ of patients and a palpable mass may be present in the pelvic area. The International Federation of Gynecology and Obstetrics (FIGO) also adapted the staging of ovarian cancer to PFTC and suggested that it is surgically staged like ovarian cancer.

Primary fallopian tube carcinomas are rare, so there are insufficient data on treatment approaches in the literature The approaches are generally determined in accordance with the data obtained from ovarian cancer. Total abdominal hysterectomy with bilateral salpingo-oophorectomy and infracolic omentectomy, appendectomy, peritoneal washing, and peritoneal biopsy constitute the primary treatment of choice for PFTC; inclusion of pelvic and para-aortic lymphadenectomy has been controversial [5].

The overall 5-year survival for patients with PFTC is 22-57\% [6]. Many prognostic factors have been investigated in an effort to better estimate patient outcome. Stage, patient age, and residual tumor after initial surgery are consistently important prognostic factors. In addition, according to some reports, a closed fimbriated end of the fallopian tube, positive peritoneal cytology, lesion site within the tube (fimbrial vs. non-fimbrial), human epidermal growth factor receptor 2/nu-positive expression, p53 alteration, elevated pretreatment cancer antigen 125 (CA-125), and lymphovascular space involvement are also important prognostic factors [6, 7]. 
In this study, we aimed to evaluate the prognostic factors and survival rates of PFTC, which is rare among gynecological cancers.

\section{Material and methods}

Thirty-eight patients with a diagnosis of PFTC were identified through the gynecologic oncology service database of our Research and Training Hospital in the period 1995-2013. Clinicopathological and surgical data were collected. All patients were evaluated for survival and disease-free survival (DFS) between the dates specified. Cases were identified according to the PFTC diagnostic criteria established by Hu et al. [8] and modified by Sedlis [9]. The following clinical data were collected from patient medical, surgical, pathological, and chemotherapy reports: demographic characteristics, presenting symptoms, serum CA-125 level, date and type of surgical procedure, presence or absence of residual tumor after surgery, number of excised and positive lymph nodes, presence or absence of ascites, tumor pathological characteristics (grade and size), type of first-line chemotherapy, date of recurrence, treatment after recurrence, date of last medical examination, and date of death. Federation of Gynecology and Obstetrics (FIGO) staging according to the 2009 revised classification system was used. Patients were classified as not having been staged if only a unilateral salpingo-oophorectomy, total hysterectomy with unilateral or bilateral salpingo-oophorectomy with or without omentectomy was performed. Partial staging was defined as pelvic washing, peritoneal biopsy, omentectomy, bilateral pelvic lymph node dissection with a unilateral salpingo-oophorectomy, or total hysterectomy with unilateral or bilateral salpingo-oophorectomy. Complete staging was defined as pelvic washing, peritoneal biopsy, omentectomy, bilateral pelvic and para-aortic lymph node dissection with a unilateral salpingo-oophorectomy, or total abdominal hysterectomy with unilateral or bilateral salpingo-oophorectomy. Optimal debulking was defined as a procedure that left a maximum residual tumor $<1 \mathrm{~cm}$ in diameter. Patients returned for a follow-up evaluation every three months for the first 2 years, every 6 months for the next 3 years, and annually thereafter. Computed tomography or magnetic resonance imaging was performed annually. The survival analysis was based on the Kaplan-Meier method, and the results were compared using the log-rank test. Progression-free survival (PFS) was defined as the time from the date of primary surgery to detection of recurrence or the latest observation. Overall survival (OS) was defined as the time from the date of primary surgery to death or the latest observation. The $\chi^{2}$ test and Student's t test for unpaired data were used for the statistical analysis. Cox regression analysis was used to determine factors affecting survival, and results are presented as hazard ratios (HRs) with 95\% confidence intervals (Cls). All statistical analyses were performed using Statistical Package for the Social Sciences (SPSS ver. 11.5). A $p$-value of $<0.05$ was considered significant.

\section{Results}

The average patient age was 55 years and ages ranged from 30 to 72 . Vaginal bleeding was observed as the most common complaint. It was observed that most of the patients were menopausal during diagnosis. $42.1 \%$ of cases were considered adnexal masses and $57.9 \%$ of them were considered ovarian cancer in the preoperative period. The distribution of CA- 125 values was $5-802 \mathrm{U} / \mathrm{ml}$ preoperatively and the distribution of tumor size was $2-14 \mathrm{~cm}$. Ascites was present in $21.8 \%$ of patients. After different surgical procedures, residual tumor was determined as $1 \mathrm{~cm}$ or less in $65.8 \%$ and more than $1 \mathrm{~cm}$ in $34.2 \%$ of patients. Sixteen patients (42.1\%) had FIGO stage I disease, 5 (13.2\%) had stage II, and 17 (44.7\%) had stage III disease. A detailed description of the operative procedures performed is shown in Table 1. Optimal surgery with residual tumor $<1 \mathrm{~cm}$ was achieved in $65.8 \%$ of cases. When the postoperative grade was examined, $78.9 \%$ of the cases were grade 3 . When postoperative pathologies were examined, $63.2 \%$ of the cases were found to be serous adenocarcinoma type and $89.5 \%$ of the patients had received adjuvant chemotherapy. Demographic and morphological characteristics of the patients are summarized in Table 1.

At patient follow-up, a significant relationship between survival probability and stage at the time of diagnosis was found (Fig. 1).

A significant relationship and correlation was found between optimal surgery and life expectancy. Better results were obtained in patients treated with optimal surgery (Fig. 2).

Survival probability was found to be higher in patients with lower CA-125 levels and serous histologic type adenocarcinoma (Figs. 3 and 4).

Survival probability was found to be higher in patients without ascites (Fig. 5).

Patients with higher grade tumors were found to have lower survival probability during follow-up (Fig. 6).

Results of univariate and multivariate analyses carried out to determine the effect of demographic characteristics and clinical features on median survival are provided in Table 2.

\section{Discussion}

Primary fallopian tube carcinoma (PFTC) is a rare neoplasm, accounting for $0.3-1.6 \%$ of all gynecological malignancies [1, 2]. The incidence of PFTC is 0.41 per 100,000 women in the United States. The incidence of PFTC varies depending on age. It is 0.02 per 100,000 young women, but the rate rises to 1.63 per 100,000 after age 70 . Unlike PFTC, a downward trend has been observed in the incidence of ovarian cancer in the US over the years [10]. There are also opinions that the real incidence may be higher due to the difficulties in diagnosis [11, 12].

In the literature, the mean age of patients with PFTC is 55 years [6]. The mean age and menopausal status of the patients in our study were in accordance with the literature. The average age of the patients in our study was 55 , while $47.4 \%$ of the patients were younger than 60 years and $52.6 \%$ were older than 60 years. Age was not found 
to be statistically significant for DFS or OS time in univariate and multivariate analyses. $73.7 \%$ of the patients were menopausal during diagnosis. Schneider et al. [13] reported that PFTC is diagnosed at an earlier stage than ovarian cancer, possibly as a result of abdominal pain resulting from tubal distention and intermittent serosanguineous discharge. During the diagnosis $73.7 \%$ of patients were in menopause. The symptoms of the patients in our study were consistent with the literature. Abnormal vaginal bleeding was detected in $21.1 \%$ of patients and $13.2 \%$ of patients complained of pain and swelling. Because PFTC usually spreads very quickly, early diagnosis of PFTC is extremely difficult. Primary fallopian tube carcinoma is usually diagnosed by operations performed for another reason. The preoperative diagnosis rate is $0.3-15 \%[14,15]$. Preoperative diagnosis is usually ovarian tumor or pelvic abscess in many patients and diagnosis is difficult before the laparotomy. The study involving 26 patients by $\mathrm{Yu}$-Jin Koo et al. showed that $65.4 \%$ of cases were diagnosed as adnexal masses, and $23.1 \%$ of them were diagnosed as hydrosalpinx preoperatively [16]. In our study, preoperatively $57.9 \%$ of the patients were diagnosed with ovarian cancer and $42.1 \%$ of them were diagnosed with adnexal masses.

There is no specific tumor marker for PFTC. However, CA-125 is often used as a tumor marker for fallopian tube carcinomas like ovarian malignancy [17]. CA-125 levels are lower in the early stages but higher at advanced stages [3]. Pre-treatment CA-125 levels are an independent prognostic factor for disease-free survival and overall survival. The median time between the increase in CA-125 levels during the follow-up and the appearance of clinical and radiological recurrences is 3 months (0.5-7 months) [15]. In our study, preoperative CA-125 levels were between and $802 \mathrm{U} / \mathrm{ml}$ and the average level of CA-125 was $41.5 \mathrm{U} / \mathrm{ml}$. In our study, there was statistically significant relationship between the CA-125 levels and the survival probability. The survival rate of the patients who had low CA-125 levels were higher at the time of diagnosis (HR: $3.7 ; 95 \% \mathrm{Cl}$ : $1.6-8.4 ; p=0.003)$. In this study CA-125 was found to be statistically significant for DFS and OS time in univariate analyses but not in multivariate analyses.

Tumor grade of the patients included in our study was consistent with the literature $[1,4] .5 .3 \%$ of patients had grade 1, 15.8\% had grade 2 , and $78.9 \%$ had grade 3 . An inverse association was found between tumor grade and survival probability (HR: $1.3 ; 95 \% \mathrm{Cl}: 0.6-2.7 ; p=0.42$ ).

In a study by E. Kalampokas and colleagues $15 \%$ of the patients had acid [6]. Our study was compatible with the literature and $21.8 \%$ of our patients had preoperative acid. When the survival rate was compared according to the presence of preoperative acid, the survival rate was higher in non-acidic patients. (HR: 3.4; 95\% Cl: 1.4-8.0; $p=0.01$ ). Presence of preoperative acid was found to be statistically significant for DFS and OS time in univariate and multivariate analyses.

During the diagnosis, the disease is a prognostic factor for the disease, and those with localized disease live longer than those with advanced disease [1]. In the studies of Rosen et al., the 5-year survival rate for stage I and stage II cases was 59\% and for stage III and IV cases was 19\% [18].
Table 1. Demographic and morphological features

$\begin{array}{lcc}\text { Parameter } & \text { No. } & \% \\ \text { Age (years) } & & \\ \leq 60 & 18 & 47.4 \\ >60 & 20 & 52.6 \\ \quad \text { Median range } & 55(30-72) & \\ \text { Menopause } & 28 & 73.7 \\ \text { Parity, median range } & 2.5(0-5) & \\ \text { Symptom } & & \\ \quad \text { Vaginal bleeding/discharge } & 8 & 21.1 \\ \quad \text { Abdominal pain } & 5 & 13.2 \\ \quad \text { Others } & 22 & 57.9 \\ \quad \text { None } & 3 & 7.9 \\ \text { Presence of ascites } & 21.8 & \\ \text { Preoperative diagnosis } & & \\ \quad \text { Adnexal mass } & 16 & 42.1 \\ \text { Ovarian malignancy } & 22 & 57.9\end{array}$

CA-125 (U/ml), median (range) $\quad 41.5$

$(5.0-802.0)$

$\begin{array}{ccc}\text { FIGO Stage } & & \\ \text { I } & 16 & 52.1 \\ \text { II } & 5 & 13.2 \\ \text { III } & 17 & 44.7 \\ \text { IV } & - & - \\ \text { Grade } & & \\ \text { I } & 2 & 5.3 \\ \text { II } & 6 & 15.8 \\ \text { III } & 30 & 78.9\end{array}$

No. of removed lymph nodes,

median (range)
Pelvic
$13.5(6-24)$
Para-aortic
$10(3-23)$

Recurrence

14

36.8

Duration of follow-up

$34.5(14-78)$

Disease-free survival

$25.5(11-76)$

Overall survival

$34.5(14-78)$

Surgical procedures

USO

$\mathrm{TAH}+\mathrm{BSO}$

2.6

$\mathrm{TAH}+\mathrm{BSO}+$ washing cytology

1

2

+ PLD + omentectomy

$\mathrm{TAH}+\mathrm{BSO}+$ washing cytology

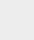

39.5

+ PPLD + omentectomy

Residual tumor at initial surgery $(\mathrm{cm})$

$\leq 1$

25

65.8

$>1$

13

34.2

Chemotherapy

Yes

89.5

No

10.5

Radiotherapy

Yes

89.5

No

38

10.5

Histological type

Serous

100

63.2

Non-serous

24

36.8

USO - unilateral salpingo-oophorectomy; TAH - total abdominal hysterectomy; $B S O$ - bilateral salpingo-oophorectomy; PLD - pelvic lymphadenectomy; $P P L D$ - pelvic and para-aortic lymphadenectomy 


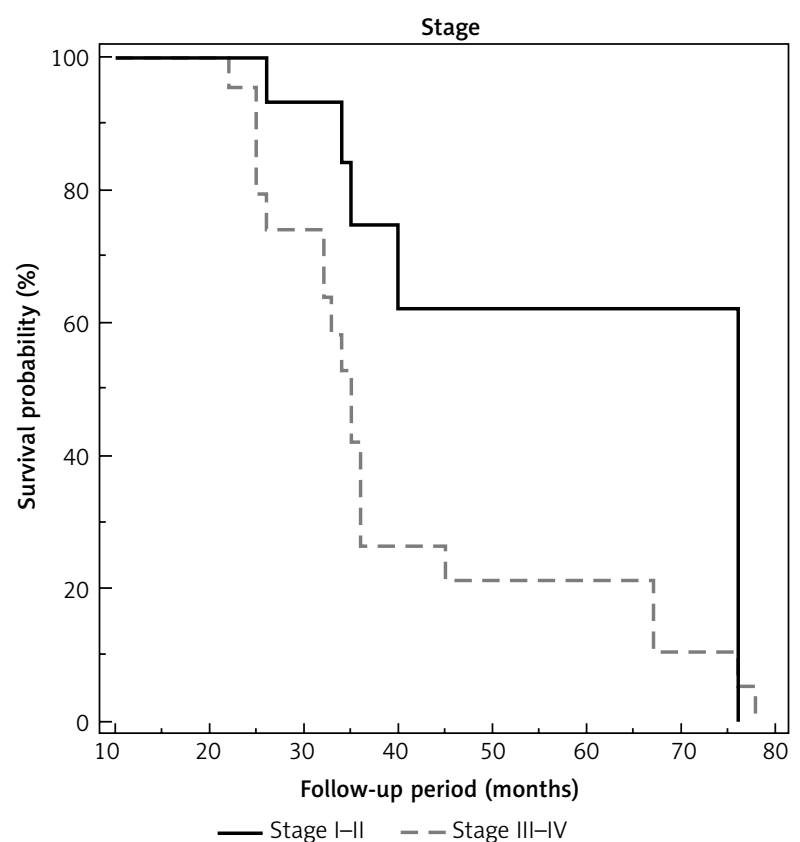

Fig. 1. Effect of stage of disease and survival probability (HR: 2.6; $95 \% \mathrm{Cl}: 1.1-5.9 ; p=0.02$ )

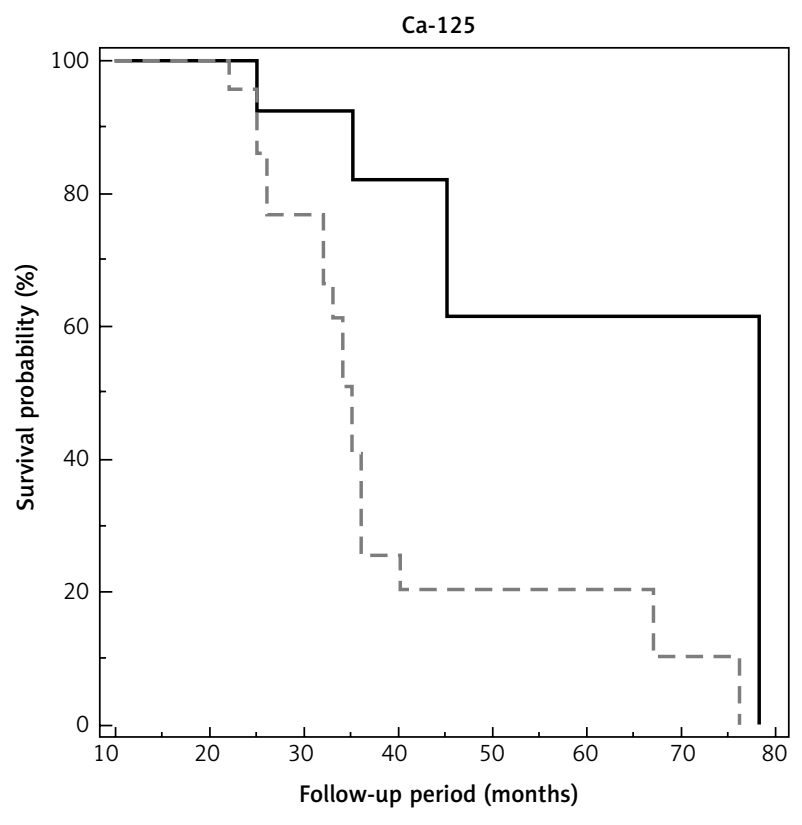

Ca-125: $-<35 \mathrm{U} / \mathrm{ml}--\geq 35 \mathrm{U} / \mathrm{ml}$

Fig. 3. Ca-125 and survival probability (HR: $3.7 ; 95 \% \mathrm{Cl}$ : $1.6-8.4$; $p=0.003)$

Our study was consistent with the literature and the relationship between stage and survival probability was found to be significant, and the survival probability of early stage patients was found to be higher (Fig. 1). Stage was found to be statistically significant for DFS and OS time in univariate and multivariate analyses.

The primary surgical principles in tubal carcinoma are as in ovarian cancer and include total abdominal hysterec-

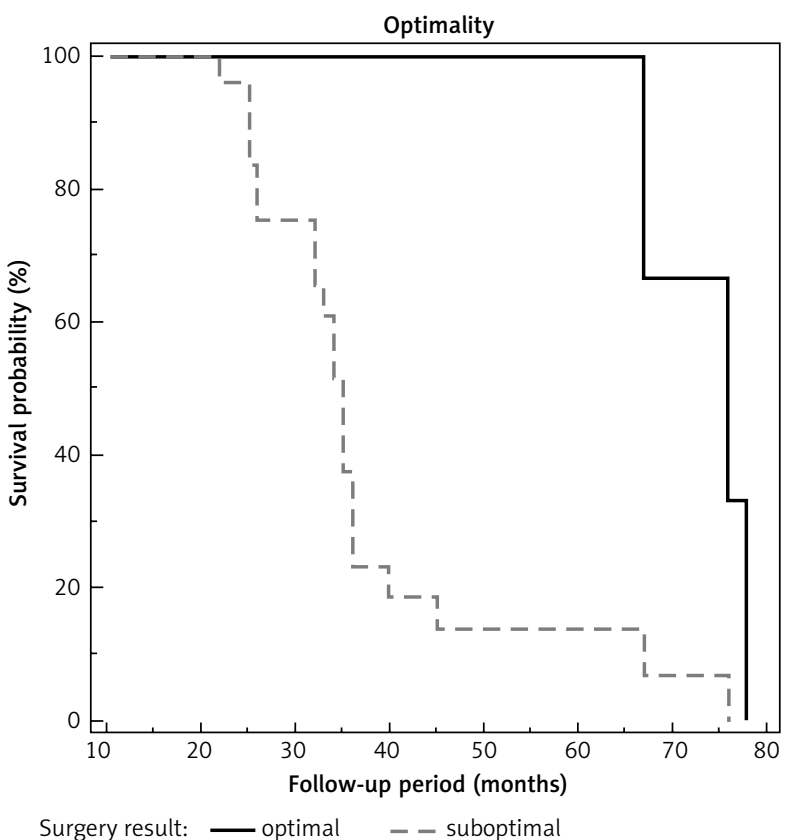

Fig. 2. Effect of optimality and survival probability (HR: $5.8 ; 95 \% \mathrm{Cl}$ : $2.6-13.0 ; p=0.0003$

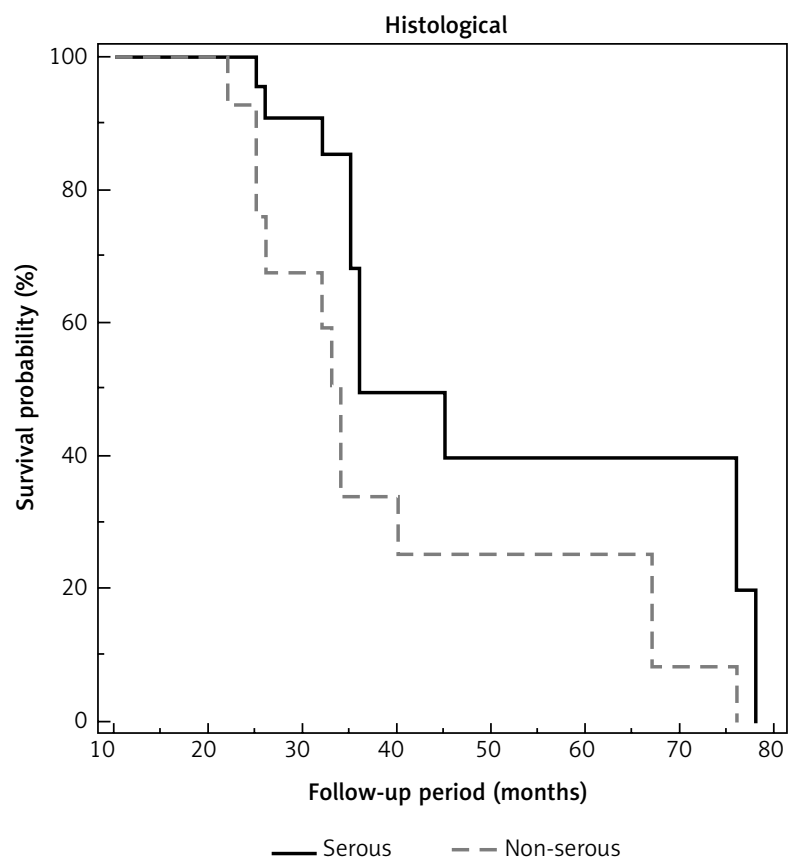

Fig. 4. Histological type and survival probability (HR: $2.1 ; 95 \% \mathrm{Cl}$ : $0.9-5.2 ; p=0.03)$

tomy, bilateral salpingoophorectomy, infracolic omentectomy and pelvic para-aortic lymphadenectomy. Peritoneal washing fluid should be taken at the beginning of laparotomy. Different surgical procedures were performed on patients in our study. Total abdominal hysterectomy and bilateral salpingo-oophorectomy was performed in 5.3\% of patients, unilateral salpingo-oophorectomy was performed in $2.6 \%$ of patients, total abdominal hysterecto- 


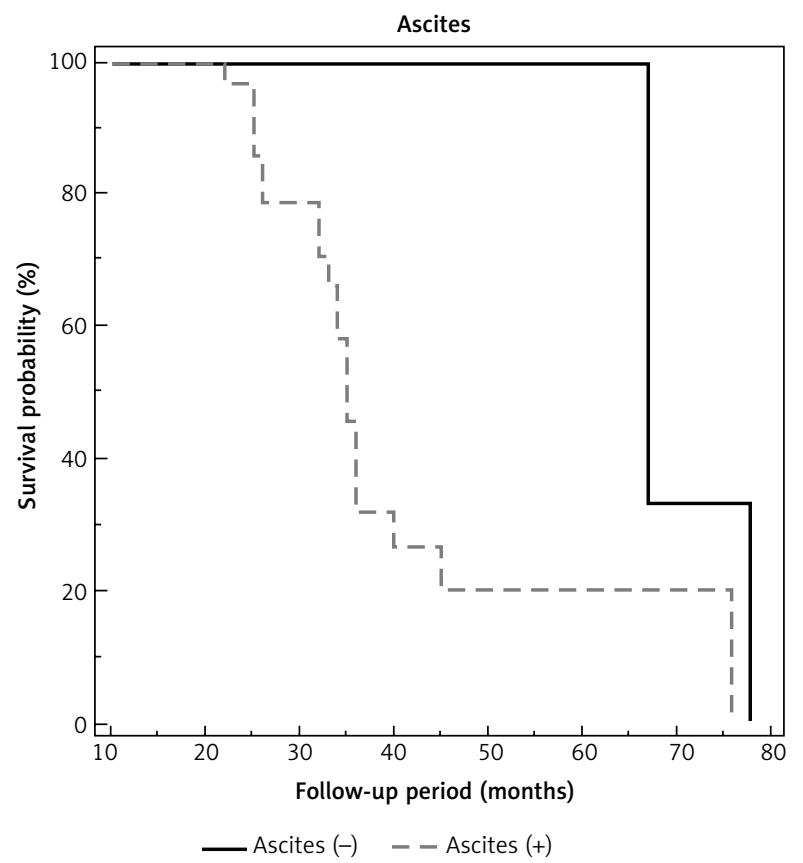

Fig. 5. Ascites and survival probability (HR: $3.4 ; 95 \% \mathrm{Cl}$ : 1.4-8.0; $p=0.01$ )

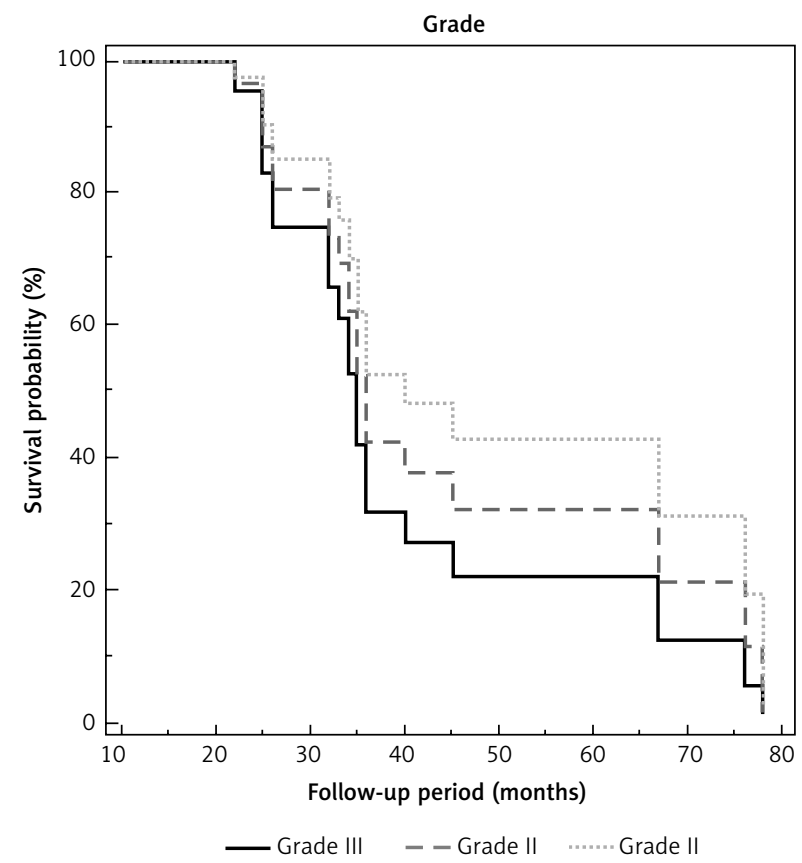

Fig. 6. Grade and survival probability (HR: $1.3 ; 95 \% \mathrm{Cl}: 0.6-2.7$; $p=0.42$ )

Table 2. Univariate and multivariate analysis of prognostic factors

\begin{tabular}{|c|c|c|c|c|c|c|}
\hline \multirow[t]{3}{*}{ Parameter } & \multicolumn{6}{|c|}{ Median survival (months) } \\
\hline & \multicolumn{3}{|c|}{ Univariate analysis } & \multicolumn{3}{|c|}{ Multivariate analysis } \\
\hline & $\begin{array}{c}\text { Hazard } \\
\text { ratio }\end{array}$ & $95 \% \mathrm{Cl}$ & $p$-value & $\begin{array}{c}\text { Hazard } \\
\text { ratio }\end{array}$ & $95 \% \mathrm{Cl}$ & $p$-value \\
\hline Age ( $<60$ years vs. $>60$ years $)$ & 1.8 & $6.2-8.1$ & 0.97 & & & \\
\hline Comorbidity (presence vs. absence) & 4.2 & $4.8-10.1$ & 0.77 & & & \\
\hline Tumor stage (I/II vs. III/IV) & 2.6 & $1.1-5.9$ & 0.02 & 3.6 & $1.6-8.7$ & 0.01 \\
\hline Tumor grade (I vs. II vs. III) & 1.3 & $0.6-2.7$ & 0.42 & & & \\
\hline Ascites (presence vs. absence) & 3.4 & $1.4-8.0$ & 0.01 & 2.1 & $1.3-5.8$ & 0.03 \\
\hline Preoperative CA-125 value (<35 U/ml vs. $35 \mathrm{U} / \mathrm{ml})$ & 3.7 & $1.6-8.4$ & 0.003 & 1.3 & $0.4-3.8$ & 0.61 \\
\hline Residual tumor size (< $1 \mathrm{~cm}$ vs. $>1 \mathrm{~cm})$ & 5.8 & $2.6-13.0$ & 0.003 & 3.2 & $1.2-5.9$ & 0.001 \\
\hline Histology (serous vs. non-serous) & 2.1 & $0.9-5.2$ & 0.003 & & & \\
\hline
\end{tabular}

$\mathrm{Cl}$ - confidence interval; CA-125 - cancer antigen 125; HR - hazard ratio

my and bilateral salpingo-oophorectomy with only pelvic lymph node dissection was performed in $39.5 \%$ of patients, total abdominal hysterectomy, and bilateral salpingooophorectomy with pelvic-para-aortic lymph node dissection was performed in $52.6 \%$ of patients. Residual tumor is an important prognostic factor after surgery. Survival of patients with remaining residue less than $1 \mathrm{~cm}$ is better so optimal cytoreduction should be performed [16]. In a study conducted in stage III-IV patients, the survival rate at 5 years was $55 \%$ for patients with a $1 \mathrm{~cm}$ or smaller residual tumor diameter and $21 \%$ for those with a larger residual diameter [4]. The median survival was 35 months in patients with residual tumor mass $<2 \mathrm{~cm}$ but $>11$ months in patients with $>2 \mathrm{~cm}$ residual tumor, and this difference was statistically significant [18]. $65.8 \%$ of patients had $<1 \mathrm{~cm}$ residual tumor but $34.2 \%$ of patients had $>1 \mathrm{~cm}$ residual tumor in our study. The survival probability of patients undergoing an optimal surgical procedure was found to be higher in this study (HR: 5.8; 95\% Cl: 2.6-13.0; $p=0.0003$ )

When the postoperative histopathologic findings of the patients were evaluated in our study, serous adenocarcinoma of $63.2 \%$ and nonserous adenocarcinoma of $36.8 \%$ were detected. In a literature review, Wethington et al. reported that $48 \%$ of patients had serous adenocarcinoma and $52 \%$ had nonserous adenocarcinoma [19]. Gadducci et al. reported that $59.1 \%$ of patients had serous adenocarcinoma and $40.9 \%$ of patients had nonserous adenocarcinoma [4]. In our study, the survival probability of patients with serous adenocarcinoma histopathology was found to be higher than the patients with non-serous adenocarcinoma his- 
topathology according to the literature when the survival probabilities of the patients were compared with the survival histopathology (HR: 2.1; 95\% Cl: 0.9-5.2; $p=0.03$ ).

In conclusion, stage is one of the factors affecting the survival probability. The pathological type of the tumor, the diameter of the residual tumor after surgery, the tumor grade, preoperative CA-125 levels and the presence of ascites were found to be factors influencing the survival probability. Among the independent factors determining the prognosis of the disease, it was found that the residual tumor amount was a factor that could change the effect. Although preoperative diagnosis of tubal carcinomas is impossible in the literature, it is observed that in the literature and in our series, a significant proportion of these patients (57.9\%) were operated on with the pre-diagnosis of ovarian carcinoma. Therefore underoing an operation by gynecological oncologists will affect the prognosis of patients. Complete staging and the amount of residual tumor less than $1 \mathrm{~cm}$ should be surgical purposes.

The authors declare no conflict of interest.

\section{References}

1. Meng ML, Gan-Gao, Scheng-Sun, Bao QC, Jung ZA. Diagnosis of primary adenocarcinoma of the fallopian tube. J Cancer Res Clin Oncol 1985; 110: 136-140.

2. Baalbaky I, Vinatier D, Leblanc E, Querleu D. Clinical aspects of primary cancer of the fallopian tube. A retrospective study of 20 cases. J Gynecol Obstet Biol Reprod (Paris) 1999; 28: 225-231.

3. Benoit MF and Hannigan EV. A 10-year reviev of primary fallopian tube cancer at a community hospital: a high association of synchronous and metachronous cancers. Int J Gynecol Cancer 2006; 16: 29-35.

4. Reyes MC, Arnold AG, Kauff ND, Levine DA, Soslow RA. Invasion patterns of metastatic high-grade serous carcinoma of ovary or fallopian tube associated with BRCA deficiency. Mod Pathol 2014; 27: 1405-1411.

5. Koo YJ, Kwon YS, Lim KT, et al. Para-aortic lymphadenectomy for primary fallopian tube cancer. Int J Gynaecol Obstet 2011; 112: 1820.

6. Kalampokas E, Kalampokas T, Tourountous I. Primary fallopian tube carcinoma. Eur J Obstet Gynecol Reprod Biol 2013; 169: 155161.

7. Bidus MA, Maxwell GL, Ros GS. Fallopian tube cancer. In: Di Saia PJ, Creasman WT (eds.). Clinical gynecologic oncology. 8th ed. Elsevier/Saunders, Philadelphia 2012; 357-368.

8. Hu CY, Taymor ML, Hertig AT. Primary carcinoma of the fallopian tube. Am J Obstet Gynecol 1950; 59: 58-67.

9. Sedlis A. Carcinoma of the fallopian tube. Surg Clin North Am 1978; 58: 121-129.

10. Stewart SL, Wike JM, Foster SL, Michaud F. The incidence of prima ry fallopian tube cancer in the Unıted States. Gynecol Oncol 2007; 107: 392-397.

11. Clayton NL, Jaaback KS, Hirschowitz L. Primary fallopian tube carcinoma - the experience of a UK cancer centre and a review of the literature. J Obstet Gynecol 2005; 25: 694-702.

12. Kone M, Body G, Calais G, Fignon A, Fetissof F, Lansac J. Primary adenocarcinoma of the fallopian tube. Retrospective study of 16 cases. Prognostic factors. J Gynecol Obstet Biol Reprod (Paris) 1992; 21: 187-192.

13. Schneider C, Wight E, Perucchini D, Haller U, Fink D. Primary carcinoma of the fallopian tube. A report of 19 cases with literature review. Eur J Gynaecol Oncol 2000; 21: 578-582.

14. Alvarado-Cabrero I, Young RH, Vamvakas EC Scully RE. Carcinoma of the fallopian tube: A clinicopathological study of 105 cases with observations on staging and prognostic factors. Gynecol Oncol 1999; 72: 367-379.

15. Ajithkumar TV, Minimole AL, John MM, Ashokkumar OS. Primary fallopian tube carcinoma. Obsest Gynecol Surv 2005; 60: 247-252.

16. Koo YJ, Im KS, Kwon YS, et al. Primary fallopian tube carcinoma: a clinicopathological analysis of a rare entity. Int J Clin Oncol 2011; 16: 45-49.

17. Puls LE, Davey DD, DePriest PD, Gallion HH, van Nagell JR Jr, Hunter JE, Pavlik EJ. Immunohistochemical staining for CA 125 in fallopian tube carcinomas. Gynecol Oncol 1993; 48: 360-363.

18. Rosen AC, Ausch C, Hafner E, Klein M, Lahousen M, Graf AH. A-15 Year Overview of Management and prognosis in primary fallopian tube carcinoma. Austrian Cooperative Study Group for fallopian tube carcinoma. Eur J Cancer 1998; 34: 1725-1729.

19. Wethington SL, Herzog TJ, Seshan VE, Bansal N, Schiff PB, Burke WM, Cohen CJ, Wright JD. Improved survival for fallopian tube cancer: a comparison of clinical characteristics and outcome for primary fallopian tube and ovarian cancer. Cancer 2008; 113: 298 306.

\section{Address for correspondence}

\section{Mehmet Kulhan}

Erzincan University School of Medicine

Basbaglar mah

24030 Erzincan, Turkey

e-mail: Mehmet_kulhan@yahoo.com

Submitted: 6.02 .2017

Accepted: $\quad 7.05 .2017$ 